\title{
Philosophiques
}

\section{Évolution et processus configurationnel chez Norbert Elias}

\section{Roger Cornu}

Volume 25, numéro 2, automne 1998

Les modèles d'évolution en économie et en sciences sociales

URI : https://id.erudit.org/iderudit/027489ar

DOI : https://doi.org/10.7202/027489ar

Aller au sommaire du numéro

Éditeur(s)

Société de philosophie du Québec

ISSN

0316-2923 (imprimé)

1492-1391 (numérique)

Découvrir la revue

Citer cet article

Cornu, R. (1998). Évolution et processus configurationnel chez Norbert Elias. Philosophiques, 25(2), 239-256. https://doi.org/10.7202/027489ar

\section{Résumé de l'article}

L'auteur montre comment $\mathrm{N}$. Elias critique à la fois la sociologie classique, qui emprunte aux sciences naturelles la théorie évolutionniste, et la sociologie moderne, qui rejette la notion d'évolution. Il analyse comment N. Elias propose une sociologie évolutionnaire à travers le concept de processus configurationnel qui abolit l'opposition classique individu-société. Dans ce cadre, la notion d'évolution appartient elle aussi au processus évolutionnaire. 


\title{
ÉVOLUTION ET PROCESSUS CONFIGURATIONNEL CHEZ NORBERT HIAS
}

\author{
PAR \\ ROGER CORnU
}

\begin{abstract}
RÉSUMÉ : L'auteur montre comment $N$. Elias critique à la fois la sociologie classique, qui emprunte aux sciences naturelles la théorie évolutionniste, et la sociologie moderne, qui rejette la notion d'évolution. Il analyse comment $N$. Elias propose une sociologie évolutionnaire à travers le concept de processus configurationnel qui abolit l'opposition classique individu-société. Dans ce cadre, la notion d'évolution appartient elle aussi au processus évolutionnaire.

ABSTRACT: The author shows how N. Elias criticizes, at one and the same time, the classical sociology using the evolutionistic theory coming from the natural sciences, and the modern sociology rejecting the notion of evolution. He analyses how $N$. Elias propounds an evolutionary sociology through the concept of configurational process which eliminates the classical individual-society opposition. In this framework the notion of evolution itself has to be seen as belonging to the evolutionary process.
\end{abstract}

Dans un entretien autobiographique, Norbert Elias, en passe d'être considéré comme un des plus grands, sinon le plus grand, sociologues du XXe siècle, explique qu'il fait régulièrement le même rêve :

Je parle au téléphone et la voix, à l'autue bout du fil me dit : a Pouvez-vous parter un peu plus fort, je ne vous entends pas ". Je me mets alors à erier, et alors la voix répète constamment : "Veuillez parler plus fort, je ne vous entends pas $[. .$. ». Certes la voix, à l'autre bout du fil, dit de temps en temps : "Je vous entends mieux à présent, un peu mieux n. Mais elle ne m'entend tout de même pas bien ${ }^{1}$.

Ce passage prend tout son sens lorsqu'on le rapproche de cette autre remarque :

Je comprends fort bien que l'on cherche ce que l'instrument que j'ai tenté d'élaborer sous la forme du concept de configuration a de commun avec les théories plus anciennes qui plaçaient les stades d'intégration collectifs des individus au-dessus des stades d'intégration individuels, par exemple avec les

1. Norbert Elias par lui-même, Paris, Fayard, 1991, p. 93. 
propositions de Durkheim et de Simmel ou avec celles des théoriciens des systèmes. Je ne peux pas apprendre aux aveugles à voir, je ne peux pas leur faire comprendre la différence. Car elle repose en dernier ressort sur un acte supplémentaire d'auto-distanciation $[\ldots]$; si l'on est incapable de procéder soimême à celte auto-distanciation, mon explication tombe dans l'oreille d'un sourd ${ }^{2}$.

Pour bien saisir la rupture à laquelle Elias nous convie, il faut nous arrêter quelques instants sur la nolion de distanciation qui est, pour lui, le fondement même de l'esprit scientifique el qui lie le développement de la pensée aux transformations sociales. Ce n'est. qu'à partiu du moment où les conditions sociales, el notamment f'urbanité, permetlent de regarder la nature, de facon exléricure, distanciée, comme un paysage, c'est-à-dire comme non immédiatement nécessaire à son existence, que les sciences de la nature peuvent naître. Il faudra attendre le moment où l'organisation sociale imposera la nécessité d'appréhender le comporlement social des autres de façon extéricure, distanciée, pour que puissent naître les sciences sociales, et ce n'est qu'à partir du moment où les relations sociales nous contraindront à dépasser la mise en scène sociale pour débusquer la personnalité réelle que pourront naître les sciences psychologiques. Ce dernier stade nous conduira vers une autoanalyse et un auto-contrôle, regroupés sous le terme d'autodistanciation. Ceci nous introduit d'emblée dans un processus évolutionnaire et fait de la notion d'évolution une notion évolutive correspondant au stade où l'on se trouve, chaque stade impliquant le stade antérieur, et au domaine à laquelle elle s'applique. Il ne peut donc y avoir de définition a priori, de norme qui régenterait les débats sur l'évolution.

L'insistance d'Elias sur la notion d'évolution et la façon dont il l'aborde constituent peut-être un des meilleur's lests que l'on puisse faire pour savoir si la sociologie d'Elias n'est qu'une poursuite de la sociologie classique, en gros celle symbolisée par Durkheim, Simmel et Weber, ou si elle est en rupture avec elle. C'est d'ailleurs autour de cette notion d'évolution qu'il situe l'incompréhension qu'il rencontre :

Je ne me dissimule pas, du reste, que l'exigence de s'attacher à l'ordre de la succession chronologique, d'étudier l'évolution progressive des groupes humains implique des difficultés particulières de communication. La notion d'évolution des sociétés est marquée actuellement d'un stigmate qui remonte à la conception prédominante de cette évolution au $\mathrm{XVIII}^{\mathrm{c}}$ et au XIX ${ }^{\mathrm{e}}$ siècle. En reprenant cette notion à la fin du $\mathrm{XX}^{\mathrm{e}}$ siècle et à la veille du $\mathrm{XXI}^{\mathrm{e}}$ siècle $[\ldots]$, on s'expose inévitablement au mépris des générations qui ont grandi avec le traumatisme de l'ancienne conception de l'évolution - l'évolution comprise comme un progrès constant de l'humanité, promettant l'accession directe à un sort meilleur ${ }^{3}$.

2. Ibid., p. 166 .

3. Norbert Elias, La société des individus, Paris, Fayard, 1987, p. 230. 
Nous pourrions résumer la pensée d'Elias de la façon suivante : ce n'est pas parce que la notion d'évolution, issue des sciences de la nature et chargée de jugements de valeurs, ne peut pas être utilisée dans les sciences de la société qu'il faut en conclure que toutes les références à l'évolution doivent être bannies. Elles se révèlent au contraire indispensables, à condition de substituer un modèle évolutionnaire et progressif à un modèle évolutionniste et progressiste.

\section{Rompre avec la sociologie évolutionniste}

\section{Individu at société}

Elias répétera sans cesse dans son œuvre que fonder la sociologie, c'est sortir la sociologie classique d'un faux débat qui fait de l'individu et de la société deux entités extérieures l'une à l'autre, les uns, comme Tarde ou Weber, mettant l'accent en priorité sur l'individu, les autres, comme Durkheim, sur la société. Cette question me semble d'autant plus importante qu'elle est au cœur de la notion d'évolution chez les classiques. Dans la façon de l'aborder, Durkheim et Tarde, dans leur débat sur le social, vont faire appel à celui qui oppose, dans le domaine de l'évolution, les tenants de Lamarck et ceux de Darwin 4 .

Discutant les théories de Spencer, Durkheim se présente comme un véritable disciple de Lamarck pour expliquer les rapports individu-société. La société est selon lui :

[...] une réalité proprement dite qui existe par soi-même et en vertu de causes spécifiques et nécessaires, qui, par conséquent, s'impose à l'homme avec sa nature propre et à lacquelle il est tenu de s'aclapter pour vivre, tout aussi bien qu'au milieu physique. [...] Aussi la société est-elle, non pas la condition secondaire, mais le facteur déterminant du progrès. Elle est une réalité qui n'est pas plus notre ceuvre que le monde extérieur et à laquelle, par conséquent, nous devons nous plier pour pouvoir vivere; et c'est parce qu'elle change que nous devons changer. Pour que le progrès s'arrêtât, il faudrait done qu'à un moment le milieu social parvint à un état stationnaire $|\ldots|^{3}$.

L'individu ne peut done être autre chose que l'intériorisation ou l'incorporation du social, les variations individuelles n'étant que le produit des variations du milieu auxquelles l'individu a été soumis. Il reste toutefois à expliquer la dynamique de la société. Ici, un lamarckisme qui expliquerait l'évolution à partir des circonstances extérieures, c'est-à-dire essentiellement physiques, comme chez

4. Pour l'analyse qui va suivre, je me suis appuyé sur : C. Bougle, Les sciences sociales en Allemagne, Paris, Felix Alcan, 1896 et La démocratie devant la science, Paris, Felix Alcan, 1903; F. Jacob, La logique du vivant, Paris, Gallimard (coll. "Bibliothècque des sciences humaines »), 1970 ; J. Piaget, Le comportement, moteur de l'évolution, Paris, Gallimard (coll. “Idées »), 1976.

5. E. Durkheim, De la division du travail social, Paris, PUF (coll. "Bibliothèque de philosophie contemporaine n), 1967 ( 1 re éd. 1895), p. 334-336. 
Montesquieu, serait contradictoire avec la règle selon laquelle le social ne peut être expliqué que par le social (proche de la formule de Lamarck : "le vivant naît du vivant "). Durkheim a alors recours à une hypothèse darwinienne qui lui permet d'expliquer l'évolution de la sociélé à partir de la densité sociale:

Si le travail se divise davantage à mesure que les sociétés deviennent plus volumineuses et plus denses, ce n'est pas parce que les circonstances extérieures y sont plus varićes, e'est que la lutte pour la vie y est plus ardente. Darwin a très justement observé que la concurrence entre deux organismes est d'autant plus vive qu'ils sont plus analogues. Ayaut les mênes besoins et poursuivant les mêmes objets, ils se trouvent partout en rivalité

C'est à coup sûr lorsqu'il pose la question de l'hérédité que la question apparait dans toule sa clarté?. La socićlé est, che\% Durkheim, une tolalité qui, prenant appui sur le biologique, cest-àdire sur la race, s'en distingue progressivement. Dans un premier temps, le social ot l'instinet se renforeent mutuellement, mais ensuite la dynamique interne de la société, en séparant celle-ei de la race, fait deelle un hépitage auquel les individus seront soumis et un organisme producteur d'individualités spécifiées. Ainsi, là où le social pouvait se transmettre, dans sa presque totalité, héréditairement, l'hérédité ne peut plus se limiter aujourd'hui qu'au type moyen, la partie la plus médiocre et la plus mécanique de l'individu. La société, par contre, va jouer le rôle d'un environnement producteur d'espèces variées.

Tarde considérera que c'est l'individu qui est le moteur de l'évolution, et ce, dira-t-il, parce que seul l'individu invente (création) et seul l'individu imite (diffusion). $\hat{A}$ ceux qui voient dans sa théorie une apologie des grands hommes et des génies, il rétorque:

Nous pourrions nous en désintéresser si le génie seul, en ec grave problème, élait en cause ; mais ce n'est pas le génie sculement, c'est notre originalité individuelle, notre génialité individuelle à nous tous, dont l'efficacité, dont l'existence même sont mises en question ; car tous, par quelque eoté, les plus obscurs comme les plus célèbres, nous inventons, nous perfectionnons, nous varions, en mème lemps que nous imitons, et il n'est pas un de nous qui ne laisse son pli, profond on imperceptible, après avoir vécu, à sa langue, à sa religion, à sa science, à son métier, à son art ${ }^{8}$.

C'est l'imitation qui va permettre de transformer un fait individuel en fait social, qui permettra la sélection et la diffusion, à l'égal d'un processus d'histoire naturelle, el c'est Darwin qui va servir ici encore de référence :

C'est pour avoir méconnu la puissance expansive de la génération, que les biologistes d'avant Darwin regardaient comme autochtones, comme nés spontanément du sol, bien des espèces semblables, parce qu'on jugeait impossible

6. Ibid., p. 248.

7. Ibid., p. 291-318.

8. G. Tarde, Études de psychologie sociale, Paris, Giard et Brière, 1898, p. 57. 
a priori, que les semences eussent franchi les steppes ou les bras de mer qui les séparaient. Donnez à la génération le temps d'agir, et il viendra un moment où une espèce aura des variétés disséminées çà et là sur le globe presque tout entier. Donnez à l'imitation le temps d'agir et il viendra un moment où une arme, un outil agricole, un procédé industriel, inventé quelqque part $|. .$.$| auront fait le tour$ du monde?

Mais pour faire le tour du monde, encore faut-il que l'invention se soit affrontée à la lutte pour l'existence reposant sur deux principes : le duel logique qui conduit à sa victoire ou à son élimination el l'umion logique qui favorise son renforcement. Nous sommes bien dans un processus de sélection des espèces et s'il y a place pour une certaine forme de lamarckisme, ce ne peut être que dans les variations des formes et des matériaux utilisés.

Rompant avec celte tradition qui, selon lui, conduit à une impasse parce qu'clle pose la société et l'individu comme extérieurs l'un à l'autre, Elias considère qu'on ne pourra aborder le problème de l'évolution qu'à partir du moment où l'on substituera une autre façon de traiter les rapports de l'individuel et du social, la vision des classiques ne faisant que dissimuler un autre débat, idéologique celui-là, celui de l'individualisme et du socialisme.

\section{Rompre avec la philosophie et l'idéologie}

Partir en chasse des mythes qui encombrent les sciences humaines est, pour Elias, une nécessité absolue, surtout dans un domaine aussi indispensable pour ces sciences que la notion d'évolution. C'est d'abord dans la philosophie mécaniste des XVII et XVIII" siècles qu'il faut aller les débusquer. Cette philosophie est marquée par le caractère immuable du monde, qu'il s'agisse de Dieu, de l'Être suprême, du grand architecte ou de la nature, et s'il y a évolution, celle-ci ne peut se faire que selon des lois qui, elles aussi, sont immuables. Seconde caractéristique de ectte philosophie, fondée essentiellement sur la physique de l'époque, la mise en valeur de l'analyse comme seule méthode scientifique pertinente. Derrière cette démarche se profile lidée que les parties ne sont pas transformées lorsqu'elles entrent en composition dans une totalité et. a contrario, que la démarche de décomposition permet de mettre en évidence les composants. On retrouve encore aujourd hui ce prineipe appliqué en socio-économie, dans les théories de décompositionrecomposition. Troisième volet de cette vision mécaniste, sa dimension atomistique qui donne la prééminence des parties sur le tout, les présentant comme des choses gardant leur substance en entrant en composition dans une totalité. Demier volet, la priorité donnée à l'analyse causale simple qui fait de l'engrenage causeconséquence un système rigide. Liée à cette analyse causale, l'obsession du commencement que l'on trouve dans tous les mythes

9. Ibid., p. 56. 
fondateurs et dont le dernier avatar serait celui du Big Bang $\grave{a}$ l'origine de l'univers. Ce qu'Elias reproche aux sciences sociales, c'est de s'être laissé piéger par cette théorie mécaniste, copiée sur la physique de l'époque et abondamment reprise par les philosophes. Une théorie de l'évolution fondée sur ces principes ne peut que nuire aux sciences sociales et doit être écartée au profit d'une analyse de l'état des sciences d'aujourd'hui et des résultats qu'clles ont fournis.

$\hat{\lambda}$ côté de ce reproche philosophique fait aux théories de l'évolution, Elias en ajoule un second : le discours idéologique, qui s'ancre sur les caractères précédents. Il y a d'abord ceux qui, comme Spengler et Toynbee, "représentent le plus souvent les phénomènes sociologiques sous la forme de cycles inéluctables qui se répètent. plus ou moins inéluctablement $|. .$.$| , considérant la société comme une$ sorte d'entité organique qui irait inćluctablement à sa mort., en passant par la jeunesse, la maturité et la vieillesse ${ }^{10}$ ". Il y a ensuite ceux qui, comme Hegel, " partent également de l'idée d'un déroulement automatique et immuable du processus social. Mais ils soulignent son caractère irrévocable dans une certaine direction. Ils croient voir $[\ldots]$ une sorte de chaine sur laquelle chaque produit approche automatiquement de son achèvement ou une voie à sens unique où tout le monde serait forcé d'avancer toujours dans la même direction ${ }^{11}$ ". Dans tous les cas, ces modèles sont dominés par le système de valeurs de ceux qui les conçoivent et qui y intègrent " un audacieux tableau général de l'évolution passée et future de l'humanité qui n'est que le reflet de leurs espoirs et de leurs angoisses $^{12}$ ».

Le drame d'Auguste Comte et de Karl Marx, c'est d'être arrivés trop tôt et d'avoir voulu créer une science sociale et une théorie de l'évolution fondées sur la recherche concrète à une époque dominée par les modèles précédents, et malgré tous leurs efforts, ils ont succombé au modèle dominant, en faisant du développement social " une projection des désirs humains de progrès constant avec, à la fin, l'attente d'une société idéale ${ }^{13}$ m. À cette valorisation de l'évolution sociale, il oppose la démarche scientifique de Darwin :

Souvenez-vous de la manière dont Darwin abordait le problème de l'évolution biologique. Il ne se préoccupait pas de savoir si les batraciens sont moralement supérieurs aux poissons, $|\ldots|$ ou les hommes plus heureux que les singes. Il se demandait seulement pourquoi et comment les diverses espèces sont devenues ce qu'elles sont à présent [...]. Le problème général de l'évolution des sociétés, et en particulier celui du temps, appelle une semblable approche ${ }^{k /}$.

10. N. Elias, La société des individus, p. 113. On trouve une analyse intéressante de la notion de jeunesse de l'humanité dans Judith Schlanger, L'énjeu et le débat. Paris, Denoël/Gonthier (coll. “ Bibliothèque Médiations "), 1979, p. 19-64.

11. Ibid., p. 114.

12. Ibid., p. 115.

13. Norbert Elias, Du temps, Paris, Fayard, 1996, p. 213.

14. Idem, p. 104. 
Cette intrusion des jugements de valeur dans la théorie a contribué à la faire rejeter par les sociologues du $\mathrm{XX}^{\mathrm{e}}$ siècle :

\begin{abstract}
Marquées des stigmates de la désillusion, il semble que les notions d'“ évolution » et de "progrès" soient devenues inutilisables par la recherche $[\ldots\}$. Les sociologues aussi se sont joints au cœur des désabusés [...]. Ils se sont cantonnés à des théories statiques de la société reposant fondamentalement sur le principe tacite qu'on ne pouvait établir une théorie universelle de la société humaine qu'à partir de l'observation de sa propre société, telle qu'elle se présentait, hic et nunc ${ }^{15}$.
\end{abstract}

Pour illustrer ce propos, on pourrait comparer deux auvres de Durkheim : De la division du travail social ot Le suicide. La première, largement marquée par la théorie de l'évolution que critique Elias, notamment dans l'analyse du passage de la solidarité mécanique à la société organique, est passée au second plan, dans la formation des sociolognes, au profit de la seconde pour sa dimension de recherche empirique hic et nunc. Face à cet abandon, Elias se révèle proche de C. W. Mills lorsqu'il écrit : "Peut-être cherche-t-on en l'occurrence à éviter le problème de l'évolution des sociétés parce que l'on risquerait de toucher à des points sensibles de la vie sociale de notre temps - des points dont le débat public est donc frappé d'un tabou social $^{16}$ n.

\title{
Pour une sociologie évolutionnaire
}

\section{L'évolution, une exigence}

Une sociologie qui exclut l'étude de l'évolution ne peut que se contenter de décrire et s'interdit de comprendre les phénomènes sociaux. La notion d'évolution est en effel plus essentielle dans les seiences sociales que dans les autres sciences, à condition qu'on la ramène à celle d'ordre successif d'évènements, en rompant à la fois avec l'introduction, dans cet ordre, de la nécessité qui conduirait inéluctablement d'une étape à une autre el de l'orientation vers un but qui donne à l'évolution, quels que soient les chemins empruntés, une fin elle aussi inéluctable. Si Elias retient quelque chose du marxisme, e'est bien celte idée que "les hommes font leur propre histoire, mais ils ne la font pas arbitrairement, dans les conditions choisies par eux, mais dans des conditions données et héritées du passé ${ }^{17}$ ", et il avance l'expression de contimum évolutif pour signilier que "dans de nombreux processus évolutifs l'unité du processus ne repose pas dans quelque substance qui demeurerait inchangée à travers tout le processus, mais dans la continuite par laquelle une certaine transformation procède d'une autre par une succession ininterrompue ${ }^{18}$. Cette succession d'étapes dans la vie sociale a un

15. N. Elias, La société des individus, p. 230-232.

16. Ibid., p. 233.

17. Karl Marx, Les luttes des classes en France (1848-1850). Le 18 brumaire de Louis Bonaparte, Paris, éd. Sociales, 1948, p. 173.

18. N. Elias, Du temps, p. 53, note 1. 
caractère d'irréversibilité, c'est-à-dire qu'il ne peut y avoir de décomposition d'un social qui réautonomiserait ses parties. La transformation ne peut que produire de nouveaux composants et une nouvelle totalité. En même temps, l'évolution peut être régressive dans la mesure oit " la modification des formations humaines est très étroitement liée à la possibilité de transmettre aux générations suivantes, sous la forme d'un savoir acquis les expériences laites par une génération donnée |...|. L'augmentation du savoir n'entraine pas de modifications génétiques de l'espèce humaine. Les expériences socialement acquises peuvent se perdre ${ }^{19}$. On pourait parter ici d'involution. Il n'en reste pas moins qu'à long terme, Elias dégage un certain nombre de grands axes d'évolution, qu'il s'agisse de l'intégration progressive des hommes dans des unités sociales de plus en plus vastes, pouvant alteindre l'humanité tout entière, du développement du procès de eivilisation et, malgré des périodes d'involution, de la monopolisation croissante de la violence, accompagnée chez les individus de processus d'auto-contrainte et d'auto-contrôle, ete.

Si l'étude de l'évolution est plus essentielle dans les sciences sociales parce que les changements sont beaucoup plus rapides que dans lordre biologique ou physicochimique, elle est aussi plus difficile parce que le chercheur est en même temps un des agents de cetre évolution. D'où la tendance à questionner une étape antérieure à partir du présent de l'observateur' en ayant recours à des valeurs hétéronomes qui vont conditionner la formulation des problèmes et le choix des documents. Ainsi :

[...] ceux qui sont déjà en possession d'un certain savoir se représentent difficilement l'expérience de ceux qui ne possèdent pas encore ce savoir [...]. On perd facilement de vue les difficultés que des générations élevées au milicu des symboles conceptuels d'un stade antérieur on éprouvé à álaborer et eomprendre les symboles de niveau fe synthèse immédiatement supérieur. [Cependant], au lien de demander à son imagination de se représenter un stade antérieur à partir d'un stade postérieur, on rlevrait plutôt s'efforeer d'accomplir l'opération inverse. Une telle perspective est à la fois plus adéguate et plus féconde $|\ldots|^{x}$.

Ce renversement de perspective implique toutefois d'être en possession d'un outillage théorique adéqual. C'est le rôle qu'Elias assigne, dans sa théorie, à la notion de configuration sociale.

\section{Le processus configurationnel}

Elias, comme nous venons de le voir, récuse des causalités simples qui feraient de l'individu ou de la société le moteur de l'évolution. Il les considère tous deux comme le produit de deux processus indissociables qui ne peuvent s'interpréter qu'à travers la

19. Norbert Elias, La société de cour, Paris, Flammarion (coll. "Champs"), 1985, p. XLVI.

20. N. Elias, Dutemps, p. 207-208. 
notion de configuration. Celle-ci prend sens à partir du moment où l'on se rappelle que ce sont les hommes qui font leur histoire, mais dans des conditions héritées du passé, c'est-à-dire que l'individu est incompréhensible en dehors des relations dans lesquelles il est intégré dès sa naissance, relations qui viennent d'être modifiées par sa naissance même. Pour faciliter la compréhension du modèle de configuration et en faire apparaître la complexité, je serai amené à standardiser un vocabulaire qui ne l'est pas toujours dans les textes d'Elias ${ }^{21}$.

Les racines de la configuration sont à rechercher dans les mutations de la pensée sociologique de l'entre-denx-guerres, notamment autour de l'idée de foule, imposée avant la Première Guerre mondiale par Tarde et Le Bon, et eelle de conscience collective introduite par Durkheim ${ }^{22}$ :

Chacun des êtres qui se croisent ainsi dans la rue, apparemment étrangers et sans relation les uns avec les autres, est, ainsi, lié par une foule de chaînes invisibles à d'autres êtres, que ce soit par des liens de travail ou de propriétés, des hens instinctifs ou affectifs. Des fonctions de l'ordre le plus divers le rendent, ou l'ont renclu, dépendant des autres et rendent, ou ont rendu, les autres dépendants de lui $[\ldots]$. Et c'est là le véritable problème : cette interdépendance fonctionnelle revêt dans chaque groupe humain une structure très spécifique ${ }^{3}$.

Interdépendance fonctionnelle est l'élément clef de la notion de configuration, le premier terme conduisant à la notion d'interrelation et le second, à celui d'interpénétration. La notion d'interdépendance est assez proche de la notion de rapport social à condition de ne pas la limiter aux rapports de production. L'individu est inséré dans un véritable entrelacs d’interdépendances, plus ou moins visibles, d'importance et d'élendue différentes et de niveaux différents, formant la structure d'une configuration à plusieurs étages. Les différents types d'interdépendances n'évoluent toutefois pas à la même vitesse, ont des durées de vie plus ou moins longues, sont plus ou moins liés entre eux, ee qui fait de la configuration un champ de tensions perpétuelles. Les interdépendances se manifestent, directement ou indirectement, à traver's les individus sous forme: d'interrelations liées aux pratiques des individus et des groupes, cellesci se structurant aussi en importance, étendue et niveaux différents ; là encore, durée de vie et évolution peuvent varier. Les interdépendanees peuvent aussi se manifester, mais pas néecssairement, à travers des liens alfectifs entre individus ou individus et choses; cel là, à nouveau, lo domaine des liens ne recouvre que

21. Ou du moins dans la traduction de ses textes. Un certain engouement actuel pour l'ceuvre d'Elias conduit à des exposés ou des utilisations plutôt simplistes de la notion de configuration. On peut en trouver un bon exemple dans l'article "Elias ^ de l'Encyclopedia Universalis.

22. Roger Cornu, "Norbert Elias et la sociologie française : l'impact de la guerre 14 18 n, Sociétés, $n^{\circ} 33,1991$, p. $227-246$.

23. N. Elias, La sociêté des individus, p. 49-50. 
partiellement celui des interdépendances et celui des interrelations, mais est essentiel à leur évolution. Ajoutons que celte configuration doit être prise comme un complexe spatio-temporel dont les limites ultimes sont, à un moment donné de l'histoire, la population de la planète pour le caractère spatial et l'histoire de l'humanité pour la dimension temporelle, tant passée que future (le futur étant représenté par une image virtuelle, l'avenir tel qu'on l'imagine).

La dimension fonctionnelle nous conduit à la notion d'interpénétration, ou au fait que les projets et les actes des agents de la vie sociale aboulissent toujours à des résultats non voulus, contrecarrés quiils sont par les projets et les actes des autres ou par la résistance des objets, et modifiés par cux. C'est done au niveau de la pratique que l'interpénétration se manifeste. On est alors sur le second axe qui permet de rendre compte de la dimension processuelle des configurations, celui des maitrises. Elias (l'ombre de Marx plane encore une fois sur lui) en distingue trois types : la maîtrise des a complexes événementiels extra-humains, c’est-à-dire ce que nous désignons par les termes vagues d'événements naturels » et. qui est grossièrement représentée par la notion de développement technologique; la maitrise "des rapports humains, que nous désignons généralement par le terme de " rapports sociaux" ", représentée par la notion de développement social ; la "maîtrise sur soi, en tant qu'individu ayant appris, dès l'enfance, à s'orienter plus ou moins seul sans pour autant être indépendant des autres ", maîtrise que l'on désigne par la notion de processus de civilisation ${ }^{24}$. Ces trois maîtrises sont elles aussi interdépendantes et évoluent chacune à son rythme propre, provoquant ainsi des tensions entre elles et des conflits entre les individus et les groupes qui coopèrent ou s'affrontent dans le cadre de ces maîtrises. Le champ de tensions se double d'un champ de conflits autour du pouvoir.

Temporalités différentes, champ de tensions, champ de conflits, c'est bien de processus configuralionne ${ }^{25}$ plutôt que de configuration dont il faudrait parler. La démarche d'Elias ressemble à l'analyse des déformations et des eatastrophes en topologie ${ }^{26}$. Rien n'est jamais stable et chaque configuration se délinit par sa capacité à absorber un

24. Norbert Elias, Qu'est-ce que la sociologie? Aix-en-Provence, Pandora, 1981, p. 192 93. Dans cet ouvrage, Elias présente un tableau assez complet de la notion de configuration mais qui ne prend tout son sens qu'à travers son ouvrage sur $L a$ société de cour et son complément indispensable, le bourgeois de cour dont il dresse le portrait dans Mozart, sociologie d'un génie (Seuil, 1991).

25. Ce terme correspond mieux aux idées d'Elias telles qu'il les développe dans ses premières œuvres et dans lesquelles il insiste sur une sociogenèse fondée sur la dynamique relationnelle. Voir Norbert Elias, La dymamique de l'Occident, Paris, Presse-Pocket, 1990, p. 98.

26. Ce point nécessiterait de plus longs développements, car on est au cour de l'épistémologie sociologique d'Elias. La réflexion topologique permet en effet d'articuler le concept d'objet et celui de processus dans un même mouvement. Voir C. P. Bruter, Topologie et perception, Paris, Maloine-Doin, 1974, p. 9-45. 
certain nombre de déformations qui seront l'origine du passage à une autre configuration sociale, et le repérage et l'analyse de ces déformations sont essentiels pour comprendre comment une configuration sociale naît de la précédente. La référence à la topologie est peut-être le meilleur moyen de faire comprendre la référence faite à Marx et les réserves qui y sont liées :

\begin{abstract}
Marx a essayć -.. dans le sillage de Hegel - de résoudre ce genre de problèmes en créant la notion de passage cle la quantité à la qualité. C'est là saus doute une acquisition conceptuelle importante ; les formulations conceptuelles de Hegel et de Marx sont des tentatives hardies d'élargir notre arsenal conceptuel pour mieux définir les nolions de continuité et de discontinuité dans les changements survenant effectivement au sein des groupements humains. Mais rien ne nous oblige à nous arrêter aux modèles que Marx et Hegel ont élaborés?
\end{abstract}

Ce qu'Elias met en exergue, e'est l'idée que chaque changement dans la quantité est en même temps changement dans la qualité (déformation) et que les sauts qualitatifs (catastrophes) sont en fait des déplacements ou des changements du centre organisateur de la configuration sociale. Ce sont les déformations qui forment la part principale du processus d'évolution, car :

[...] toutes les modifications s'opèrent toujours très lentement pendant un long espace de temps, à petits pas imperceptibles pour nos oreilles attentives aux seuls évènements qui font du bruit. Les grandes explosions qui transforment brusquement l'existence et l'attitude des individus et qui, pour cette raison même s'imposent à notre perception, ne sont que des phénomènes partiels s'inscrivant. dans la ligne progressive el presque imperceptible des changements sociaux $[\ldots]^{3}$.

Par contre, si l'on veut trouver un "début s à une configuration, e'est dans les catastrophes qu'il faut aller le chercher, mais ce ne sera toujours qu'un début relatif. La rupture avec la sociologie classique est ici évidente, car elle évacue la distinction entre le normal el le pathologique, élément elef de la sociologie durkheimienne, ou la catégorie de dysfonctionnement des sociologies hic et munc.

La notion de processus est encore importante dans la mesure où Elias pense en termes de chaînes d'interdépendances sur lesquelles se construisent des configurations partielles et les relations entre ces configurations. Globalement, on a toujours affaire à un double mouvement de différenciation el d'intégration, l'une présupposant. l'autre. Niveau de diflérenciation et niveau d'intégration seront ainsi deux éléments clefs de l'analyse de l'évolution.

Si l'on considère les différents éléments du processus configurationnel, tel que je viens de le dessiner à grands traits, une conclusion s'impose : seule la recherche concrète peut permettre de comprendre le passage d'une configuration à une autre, la sélection qui se fait dans le champ des possibles. Une théorie qui ne

27. N. Elias, La société de cour, p. 263.

28. N. Elias, La dynamique de l'Occident, p. 229-30. 
s'appuierait pas sur les faits ne pourrait être qu'une construction idéologique abstraite, mais a contrario aucune recherche concrète ne peut aboutir sans schéma théorique. Ce sont deux processus inséparables.

\section{Le rôle de la subjectivité}

Si les hommes font leur propre histoire, cest que leur subjectivité est un moment el du processus historique el de l'inferprélation qüils se donnent de ce processus, celte interprélation intervenant dans les pratiques mêmes des agents sociaux, et done dans l’évolution. Une configuration qui est la souree de la configuration suivante determine à la fois celle nouvelle configuration et la façon dont celle configuration est perçue par les individus (sous cette appellation, entendons les individus et les groupes, comme en statistique où les groupes peuvent être considérés comme des individus) qu"elle intègre. Mais en même temps, la nouvelle configuration détermine la façon dont les individus jugent la configuration antérieure et la façon dont ee jugement conditionne leur vision de la configuration à laquelle ils appartienment ${ }^{(2)}$. II faut ajouter ici que les configurations antéricures ne sont pas néeessairement des conligurations disparues de la surface de la terre. Certaines configurations actuelles, auxquelles nous sommes aujourdhui reliés, présentent des traits analogues à des configurations antérieures à la nôtre, ce qui renforee l'idée que les jugements sur le passé sont aussi des jugements sur le présent, notamment sur les pays sous-développés :

Des expressions comme "l'homme des eavernes ", l'" homme de l'âge de pierre ", le "primitif" ou encore le "sauvage " montrent bien la distance que l'on établit artificiellement entre soi-même et ces autres hommes et le mépris non négligeable avec lequel on considère habituellement du haut d'un savoir étendu el de la domination qu'il pernel d'exercer sur eux, la plupart des représentants de ces starles anciens qui vivent encore aujourd'hui. Il n'y a pas d'autre justification à cette distance et à ce mépris que l'orgueil imétléchi qu'ils exprinento

Cette reconnaissance de l'articulation d'une configuration sur l'autre dans le temps, ou d'une dépendance de l'une par rapport à l'autre dans lespace, et leur examen seicntifique sont indispensables:

[... car on ne contribue pas seulement à une meilleure compréhension du développement des groupes sociaux, dont l'aboutissement a ćté notre réseau d'interdépendances actuel, mais on découvre aussi dans les formations sociales qui nous paraissent aussi étranges que les individus dont elles étaient constituées, des aspects essentiels grâce auxquels nous sommes à même de nous mettre à la

29. Norbert Elias par lui-même, p. 127, note

30. N. Elias, La société des individus, p. 224. 
place des personnes dont le mode de vie ne ressemble pas au nôtre, qui font partie d'autres sociétés et se signalent par des caractères propres ${ }^{31}$.

Sur ce point, Elias s'oppose violemment à Lévi-Strauss et a à son évident ressentiment contre tous les concepts évolutionnistes ". Là où Lévi-Strauss craint que la démarche évolutionnaire porte atteinte à la dignité de la a pensée sauvage », Elias considère, au contraire, que " c'est seulement en comprenant et en expliquant la pensée et l'expérience des hommes aux stades antérieur's comme caractéristiques de leur position dans un ordre de succession que l'on peut parvenir à comprendre et à expliquer la pensée et l'expérience des groupes qui représentent un stade ultérieur ${ }^{32}$. En allant sur cette voie :

[...] on atteint un autre plan, où l'altérité dles autres sociétés et des hommes qui les constituent n'est pas ressentic comme une chose bizarre, où les hommes des autres sociétés peuvent devenir les objets de notre savoir ot de notre compréhension. Une recherehe sociologique ou historique purement descriptive s'arrête -- pour employer une autre image -- sur la ligne à partir de lacuelle les hommes qu'on prétend étudier nous apparaissent pour ainsi dire à la troisième personne. C'est seulement en poussant l'étude qu'on reconnaît dans l'étude un être humain, qu'on arrive à l'intégrer dans notre vision, à l'inclure dans notre " nous n, à le comprendre vraiment.

\section{Et le point central ici, c'est que :}

[...] en découvrant des interdépendances, on rétablit l'ultime identité de tous les hommes, identité sans laquelle toute relation humaine, même celle qui s'établit entre le chercheur et l'objet de sa recherche, entre les vivants et les morts, retombe au niveau de la barbarie, de l'épocjue reculée et sauvage, où l'individu appartenant à une autre société élait considéré comme un être étrange el souvent mềne pas lumain".

Ces eitations un peu longues mais nécessaires font apparaître clairement la profondeur à laquelle Elias situe la notion d'évolution dans la pensée scientifique. II considère que le chercheur, à la fois, est engagé dans la société dans laquelle il vit et doit se distancier par rapport à clle et à son objet d'étude. Avec la prise en compte de l'évolution, recherche scientifique et humanisme se rejoignent $t^{34}$.

31. N. Elias, La société de com: p. 2314.

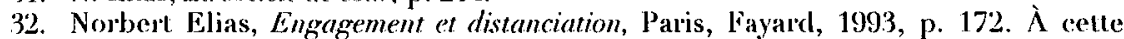
occasion, Elias demande, à juste titre, que l'on réévalue l'ouvre de Lévy-Bruhl, injustement mise à l'écart.

33. N. Elias, La société de cour. p. 235.

34. On retrouve ici les éléments d'un débat des années 1960 autour clu marxisme et du structuralisme. La lecture d'Elias fait apparaître que ce n'était pas le marxisme, comme l'affirmait Althusser, mais l'approche structuraliste du marxisme qui était un "anti-humanisme théorique ". 


\section{Retour vers la "grande évolution"}

\section{La synthèse pogressive}

Pour aller plus loin dans une approche évolutionnaire du social, la notion d'évolution doit être précisée en dépassant la division actuelle des sciences, si on veut y voir clair et éviter d'interpréter le social avec des modèles physicochimiques on biologiques. Toutefois, il faut considérer l'ensemble dans un modèle global d'évolution sans confondre deux ordres de faits, ou deux ordres de questionnement. Le premier ordere porte sur la formation de l'univers, l'apparition du vivant, puis celle de l'homme social. On est là dans un ordre emboîté en paliers d'intégration suceessifs qui n'élimine pas le palier antérieur, mais où chaque étape présuppose le palier antérieur. Chaque palier est, par rapport au précédent, différenciation et intégration. Dans celte analyse, Elias allirc l'attention sur ces deux concepts déjà rencontrés autour du processus configurationnel, comme concepts nécessaires à l'étude de l'évolution sociale, concepts que l'on retrouve aussi chez F. Jacob au sujet de l'évolution biologique. Ces remarques sur la grande évolution conduisent Elias à rejeter, comme il l'avait fait pour la dichotomie individu/société, la dichotomie nature/société (ou homme/nature) et la notion d'environnement, l'homme étant un élément du palier physicochimique et du palier biologique, aussi bien que du palier social. Un sociologue se doit done d'intégrer la mature dans le processus configurationnel, pour autant qu'elle concerne le social, ne serait-ce que comme réservoir de ressources ou territoire.

Elias affirme en même temps que chaque palier de la grande évolution a ses formes propres d'évolution, de différenciation et d'intégration, et que le tort de l'ćvolutionnisme était d'appliquer les formes de l'un des palier's à l'autre. Dans les processus d'intégration physicochimique, par exemple, un processus de désintégration peut permettre de retrouver les parties constituantes, ce qui n'est pas le cas dans le social. Les rythmes mêmes d'évolution sont différents d'un palier à l'autre, comme nous l'avons vu dans les différents paliers d'une configuration. Ceci implique que l'évolution, dans les différentes sciences, n'a pas la même importance et qu'elic $y$ a des formes différentes ${ }^{35}$, ce qui conditionne le travail du chercheur. Un chimiste ou un physicien, par exemple, peut faire des expériences à des périodes très éloignées les unes des autres sans que cela influence notablement les résultats, tandis que le sociologue ne pourra jamais faire deux fois la même observation. En prenant toutefois en compte l'évolution spécifique de chacun des paliers, les sciences se sont rapprochées sur un autre plan - et Elias prend ici

35. Pour l'histoire des débats sur l'évolution en biologie, voir F. Jacob, La logique du vivant. Sur les débats actuels sur la façon d'aborder la question de l'évolution, voir P. Tassy (dir.), L'ordre et la diversité du vivant, Paris, Fayard (coll. "Fondation Diderot $n), 1986$. 
l'exemple de la cosmologie - , celui des processus génétiques. La cosmologie est exemplaire puisque de science statique étudiant les rapports entre les planètes, on est passé à l'analyse de la genèse de l'univers. À côté de la démarche génétique, et pour la fonder, s'est imposée une démarche de type archéologique et paléontologique ${ }^{36}$.

Tous ces éléments de convergence auraient dô, selon Elias, conduire à la construction d'une représentation commune et arliculée de l'évolution et à une transformation du système des sciences, et pourtant il n'en est rien. Et de noter:

Il y a des situations dans le savoir humain où certains problèmes demeurent insolubles parce que les faits dont on aurait besoin pour les résoudre ne sont pas encore connus dans l'état actuel du savoir. Il y en a d'autres dans lesquelles le fond social de savoir contient à profusion les faits indispensables à la solution du problème, mais où les manières de pensée dominantes, les eatégories et concepts en place bloquent la voie de la solution. Nous sommes ici en présence de ce second type de frein".

\section{La synthèse symbolique}

Pour comprendre le décalage entre la collecte des faits indispensables et la mise en place d'un schéma d'interprétation, il faut prendre en compte le double aspect de la notion d'évolution: l'évolution comme ordre chronologique de processus événementiels et l'évolution comme modèle d'interprétation ; c'est au premier' sens que renvoie la synthèse progressive et au second, la synthèse symbolique. Il faut toutefois ajouter que la synthèse progressive ne peut être atteinte qu’à travers la synthèse symbolique ${ }^{38}$. D'où l'importance de l'étude de cette dernière qui doit elle aussi être soumise à la notion d'évolution. La naissance des sciences et leur ćvolution sont partic intégrante de l'évolution sociale, ce qui nécessite une élude de l'évolution scientifique intégrée dans une étude de l'évolution sociale. C'est une préoceupation constante dans lexuvre d'Elias. De ce point de vue, les scienees de la nature sont plus importantes que les sciences humaines pour découvrir la difference entre synthèse progressive ef synthèse symbolique. L'évolution des phénomènes naturels étant beaucoup plus lente que celle des phénomènes sociaux, on relève, dans l'histoire des sciences, des interprétations complètement différentes de faits qui, du point de vue de l'histoire humaine, peuvent être considérés comme stables. Ces variations d'interprétation, que l'on aurait pu penser fondées uniquement sur linsuffisance des faits collectés, relèvent, pour une part, de la dimension sociale de l'activité scientifique et ne peuvent etre comprises sans le recours aux sciences humaines.

36. H. Delporte, Archéologie et réalité, Paris, éd. Picard, p. 7-22.

37. N. Elias, Engagement et distanciation, p. 193.

38. La séparation entre synthèse progressive et synthèse symbolique pourrait être rapprochée du débat des années 1960 entre concret réel et concret pensé. 
Le chercheur est engagé dans sa société avant d'être engagé dans son activité scientifique, et est donc soumis d'abord à des concepts d'expérience qui traduisent une certaine façon de "vivie" les séquences d'événements. La notion de concept d'expérience doit être rapprochée d'un débat amorcé à la fin des années 1930 par un texte d'Einstein sur les concepts scientifiques. Celui-ci partait en effet de l'idée que "toute la science n'est rien de plus qu'un raffinement de la pensée de tous les jours. C'est pour cette raison que la réflexion eritique du physicien ne peut pas se limiter à l'examen des eoncepts de son domaine propre. Bien au contraire, il ne peut rester indifférent à une réflexion erilique sur la pensée de lous les jours, qui est bien phus difficile à analyser ${ }^{30}$ ". Il appelait concepts primaires des concepts qui sont "directement el intuitivement reliés à des complexes typiques d'expériences sensibles" ". C'est sur la hase de ces concepts primaires, et par palier's ou couches suecessives de concepts que se construit la pensée seientifique. Celle-ci implique donc ces concepts primaires, mais s'en distancie progressivement tout en leur restant liée. On peut aisément voir le lien entre cette conception et les idées d'Elias à travers ses références à l'oruvre de Lévy-Bruhl qui fait ouvertement référence à Einstein, notamment dans l'analyse des deux fonctions des concepts, dont l'une est essentiellement tournée vers la pratique et l'autre, ver's l'interprétation du monde, fût-elle scientifique ou mythique $e^{41}$.

Sous l'appellation de concept primaire, de concept à fonction pratique ou de concept d'expérience se cachent des phénomènes complexes. C'est d'abord l'utilisation de termes anthropomorphiques ou sociomorphiques pour désigner des phénomènes du domaine biologique ou physicochimique. Il suffit de penser ici à l'image de la division du travail pour parler du corps humain, à l'emprunt de la lutte pour l'existence fait à la pensée ćconomique de Malthus par Darwin pour traiter des espèces animales, ou au termo de société pour désigner certains phénomènes d'organisation collective notés chez les animaux. À l'inverse, les concepts d'expérience peuvent partir de l'élat des sciences à un moment donné et de l'expérience accumulée dans un domaine et qui sera transférée dans un autre. C'est le cas des notions d'organe et d'organisme, issues de la biologic et transférées en sciences sociales. Un troisième domaine concerne les termes de la vie courante qui peuvent se révéler ambigus. Elias en donne un exemple lié à la théorie de l'évolution : Iorsqu'on dit qu'un nouveau palier d'intégration se forme "à partir de |... , ce qui est problématique, c'est de savoir à quoi se rapporte l'expression "à

39. Albert Einstein, Conceptions scientifiques, Paris, Flammarion (coll. "Champs "), 1990, p. 21.

40. Ibid., p. 25.

41. Lucien Lévy-Bruhl, Les carnets de Lucien Léry-Bruhl, Paris, PUF, 1949, p. 224 et ss. H. Wallon, à son tour discutera les idées de Lévy-Bruhl dans De l'acte à la pensée, Paris, Flammarion (coll. “ Champs »), 1970, p. 73 et ss. 
partir de ". Elle peut signifier " se compose de " ou " se développe à partir de ${ }^{{ }_{42}}$ . On trouve un élément similaire chez Einstein lorsqu'il note :

[... que, du point de vue de l'expérience courante, la signification de la simultanéité d'événements distants dans l'espace est a priori claire, et par consécquent, aussi en physique - cette illusion a son origine dans le fait que, dans notre expérience quotidienne, nous pouvons négliger le temps de la propagation de la lumière. Nous sommes, pour cette raison, habitués à ne pas faire de distinction entre ce qui est "simultanément vu" et ce qui "arrive simulanément "; le résuliat en est que la différence entre le temps et le temps local s'elface ${ }^{\text {i⿱ }}$.

De cette ambigüté, le scientifique, pas plus que le philosophe, ne peut se départir complètement, ce qui a fait dire à Judith Schlanger que l'on " pense toujours la bouche pleine ${ }^{\text {í }}$ ", car aucun concept ne peut se libérer complètement des concepts primaires.

L'étude de l'évolution ne peut être séparée de la notion de temps qui s'y trouve impliquée. Elias met en place, à travers celle-ci, la catégorie de concept d'expérience opposée à celle de concept structurel, la seconde impliquant une distanciation suffisante pour qu'elle puisse être considérée comme une catégorie de l'évolution pro-gressive. C'est entre ces deux univers conceptuels qu'Elias situe la différence entre l'histoire et la sociologie. La première en effet, pour autant qu'elle reste une histoire factuelle, se situe sur une vision du temps qui sépare " hicr, aujourd'hui et demain „, ou " passé, présent, futur" qui sont des catégories de l'expérience quotidienne ${ }^{45}$ et non des catégories capables d'analyser des processus qui s'interprètent en termes de "avant et après "ou "antérieur et postérieur ", celles-ci relevant de l'évolution progressive dans laquelle un élément d'hier peut être aussi un élément d'aujourd'hui et de demain ${ }^{\text {í }}$. Pour qu'il y ait réellement saisie de l'évolution, il est nécessaire que le continuum de référence soit le plus éloigné possible de l'expérience humaine el qu'il puisse être commun à lous les paliers de l'évolution.

La lechure de l'oeuvre d'Elias fait bien apparaître une rupture par rapport aux conceptions sociologiques antépieures en ce quil refuse les polarités substantialistes individu/société et nature/société ou culture. Rupture aussi dans le fail de voir dans l'évolution de l'humanité a un fleuve avec trois courants d'évolution différents", l'évolution biologique, l'évolution sociale et l'évolution historique, qui se structurent dans le processus configurationnel. Rupture encore dans la façon de replacer les constructions scientifiques dans le courant de l'évolution à travers la notion d'évolution symbolique.

42. N. Elias, Engagement et distanciation, p. 218.

43. A. Einstein, Conceptions scientifiques, p. 35.

44. J. Schlanger, L'enjeu et le débat, p. 7.17.

45. Ibid., p. $95-118$.

46. N. Elias, Dutemps, p. 90-94. 
Rupture enfin dans sa façon de rejeter aussi bien les visions pessimistes de l'évolution fondées sur la mort des civilisations et l'éternel retour que celles fondées sur une idée béate du progrès. Dans sa façon de s'engager autour de sa nolion de sociologie évolutionnaire et de refuser l'idée qu'une sociologie du hic et nunc rejetant la notion d'évolution puisse réllement permettre la compréhension de notre monde, Elias prône une certaine responsabilité du travail intellectuel et, traitant des aspects régressif's liés à l'intégration progressive des létats, il nole: "Certains de mes lecteurs souhaiteraient peut-être ne me voir évoquer iei que des aspects positifs et réjouissants de l'histoire de l'humanité. Mais une telle sélection correspondrait très exactement à ce qu’il faut entendre par la trahison des cleres ${ }^{17}$ w. La prise en comple de l'évolution et la l'açon de structurer le processus configurationnel conduisent enlün Elias à s"interroger sur le rôle de la division actuelle des disciplines scientifiques dans le relard pris par les modèles dinterprétation sur le travail empirique, el à prôner, sinon un redécoupage du champ scientifique, du moins une mulliplication des interprétations interdisciplinaires, sa formation de médecin n'étant sans doute pas étrangère à cette façon d'envisager les choses.

Département d'ergologie

Université de Provence

47. N. Elias, La société des individus, p. 219-220. 